\title{
A virtual mass model in train aerodynamics
}

\author{
I. Tanackov ${ }^{1,2}$, J. Tepić ${ }^{1}$, M. Kostelac ${ }^{3}$, G. Stojić ${ }^{1} \&$ Z. Masal ${ }^{2}$ \\ ${ }^{I}$ Faculty of Technical Sciences, University of Novi Sad, Serbia \\ ${ }^{2}$ Serbian Railways, Beograd, Serbia \\ ${ }^{3}$ Faculty of Mechanical Engineering and Naval Architecture, \\ University of Zagreb, Croatia
}

\begin{abstract}
The paper presents a new approach to the research into the aerodynamic characteristics of vehicles and their interpretation. The approach is based on the theory of collision. The virtual mass is related to the unit coefficient of restitution of a central collision and to the absorption of kinetic energy. The basic system is chosen to be a train, and the system involving the virtual mass effect is air. A series of successive collisions of these two systems provides a model of air resistance. The expression derived for the unit virtual mass states that it is equal to a half of the coefficient of the square polynomial term of the air drag function. Thus, a physical interpretation of the virtual mass has been obtained and the metric system of the air resistance has been determined. A relation between the virtual mass and the most important aerodynamic characteristic, i.e. the coefficient of aerodynamic profile, has been established.

Keywords: aerodynamics, train resistance, ETR 500.
\end{abstract}

\section{Introduction}

All vehicles move in two systems. The first is the ground, which acts on the vehicle in an indirect way through gravity. The product of mass of the vehicle and the local gravity gives its weight, which is directly related to the resistance to motion. A complex structure of resistance is of particular importance in railway vehicles.

The other material system is the surrounding air which acts on a land vehicle directly, according to Newton's third law, i.e. the action-reaction law. The motion of a vehicle changes the inertial status of elements of the material system of air, resulting in a reactive natural phenomenon known as air resistance. 
Bearing in mind the intensity of the vehicle exploitation, reduction in the resistance to motion of the vehicle is the basis for efficient energy use. Therefore, it is very important to accurately calculate the resistance to motion of a vehicle. The solution to the problem of the magnitude of air resistance related to high-speed trains is of particular importance. It has been determined experimentally that in the cases when high speeds are involved the air resistance forms a major part in the resistance to motion of a vehicle [1]. The problem of air resistance is particularly pronounced with trains due to their length, i.e. their large side surfaces. One of the means to reduce air resistance is the formation of the aerodynamic profile of the train, with special attention paid to the aerodynamic shape of the front, which plays a major role in the total train resistance [2-5].

The problem of air resistance becomes even more complex when it comes to different limitations related to the volume [6-9] or to the motion of air masses and wind [10-15].

\section{Theory of virtual mass}

When a moving material system comes into contact with another material system which can be moving or at rest, a complex dynamic process in the form of collision arises. Two material systems collide if at least one material system has a certain amount of motion, i.e. kinetic energy. After the collision, changes in the speed of the material systems occur. These changes include changes in the direction, sense and intensity.

A central collision of two systems occurs when both systems move on a straight line connecting their centres of inertia. If the contact point lies in the plane that connects the centres of inertia of the systems, there is no change in their direction of motion.

An elastic collision implies that the geometric characteristics of the systems before and after the collision remain unchanged. In real conditions, the energy balance of the kinetic energy of the systems before the elastic collision is not equivalent to the kinetic energy of the systems after the elastic collision.

The following is assumed about the virtual mass:

1. Virtual mass in a central collision, and

2. Virtual mass in a perfectly elastic collision.

A body of mass $m$ has a velocity $v_{0}$, and a body of mass $m^{\prime}$ has a velocity $v_{0}^{\prime}$. Both bodies move on a straight line connecting their centres of inertia. The postcollision velocity of the body of mass $m$ is $v_{1}$, and of the body of mass $m^{\prime}$ it is $v^{\prime}{ }_{1}$. According to the theorem on the change of momentum, it follows that:

$$
m v_{0} \pm m^{\prime} v_{0}^{\prime}=m v_{1}+m^{\prime} v_{1}^{\prime}
$$

A central collision between two bodies (Fig. 1) can occur:

a) when two bodies are moving along the same line and in the same direction with the velocities which enable the collision;

b) when two bodies move along the same line, but come from opposite directions; this is a head-on collision. 
c) when one body is at rest on the line along which the other body is moving towards that body at rest.

The sign " \pm " from equation (1) refers to the cases defined under a) and b), while in the case defined under c) the starting velocity of $m^{\prime}$ is equal to 0 and the whole term is not considered. The second equation for determining the velocities $v_{1}$ and $v_{1}^{\prime}$ is obtained from Newton's Law of Restitution which defines the coefficient of restitution as:

$$
\kappa=\frac{v_{1}^{\prime}-v_{1}}{v_{0} \pm v_{0}^{\prime}}
$$

By inserting expression (2) into expression (1), the following expressions for the post-impact velocity are obtained:

$$
v_{1}=\frac{m v_{0}-\kappa m^{\prime} v_{0} \pm(\kappa+1) m^{\prime} v_{0}^{\prime}}{m+m^{\prime}}, v_{1}^{\prime}=\frac{ \pm m^{\prime} v_{0}^{\prime} \pm \kappa m v_{0}^{\prime}-(\kappa+1) m v_{0}}{m+m^{\prime}}
$$

In the case of a perfectly elastic collision, the coefficient of restitution equals $1(\kappa=1)$. The simplest case is when $v_{0}^{\prime}=0$ and then expressions from (3) can be written as:

$$
v_{1}=\frac{\left(m-m^{\prime}\right) v_{0}}{m+m^{\prime}}, v_{1}^{\prime}=\frac{2 m v_{0}}{m+m^{\prime}}
$$
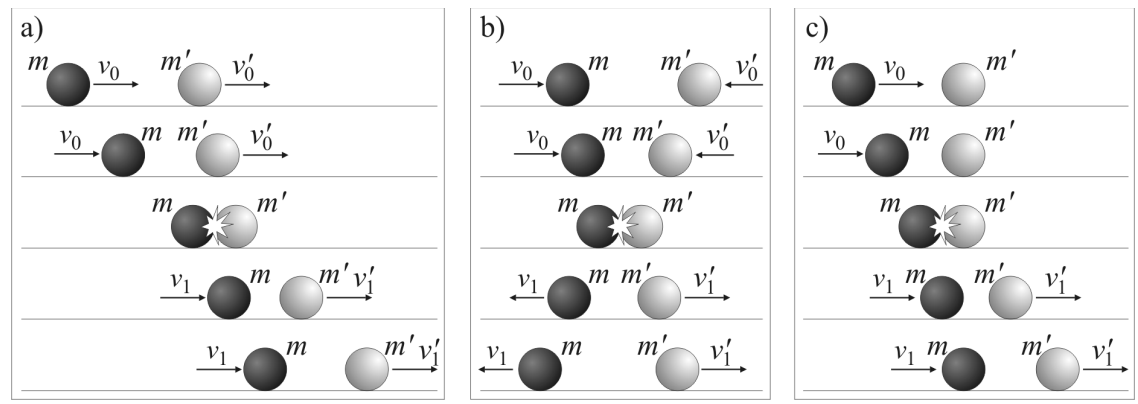

Figure 1: Central collisions: (a) rear-end collision (b) head-on collision (c) incursion collision.

If the virtual mass model is applied, the equivalence between a change in the vehicle speed (a change in the momentum, a change in kinetic energy) and the perfectly elastic central collision is established. Thus, the action of resistance forces can be indirectly expressed by a change in momentum.

$$
\Delta K=m\left(v_{0}-v_{1}\right)=m v_{1}^{\prime}
$$

Therefore, the third characteristic of virtual mass has to be introduced: after a collision has happened or after any change in the inertia, the virtual mass disappears. The virtual mass behaves exclusively as an absorber of kinetic energy (of the momentum) and it is impossible for virtual mass to take part in a chain reaction of the collision with other virtual masses. 
For such a system, the law of conservation of kinetic energy is valid. A body of mass $\mathrm{m}$ can be stopped only in a collision with a body of the same or greater mass. Provided that $m>m^{\prime}$, the absorbed kinetic energy of virtual masses from (4) result in a geometric sequence. The sum of absorbed kinetic energies of virtual masses at unit spacing is equal to the initial kinetic energy. The condition $m>m^{\prime}$ is always fulfilled when the virtual mass model is applied in aerodynamics. The vehicle mass $m$ is always greater than the air mass $\mathrm{m}^{\prime}$.

$$
\begin{aligned}
\sum_{i=0}^{\infty} \frac{m^{\prime} v_{i}^{2}}{2} & =\sum_{i=0}^{\infty} \frac{m^{\prime} v_{i}^{2} \frac{4 m^{2}}{\left(m+m^{\prime}\right)^{2}}}{2}=\frac{2 m^{2} m^{\prime} v_{0}^{2}}{\left(m+m^{\prime}\right)^{2}} \sum_{i=0}^{\infty}\left(\frac{m-m^{\prime}}{m+m^{\prime}}\right)^{2 i}= \\
& =\frac{2 m^{2} m^{\prime} v_{0}^{2}}{\left(m+m^{\prime}\right)^{2}} \cdot \frac{\left(m+m^{\prime}\right)^{2}}{4 m \cdot m^{\prime}}=\frac{m v_{0}^{2}}{2}
\end{aligned}
$$

\section{Functions of the resistance force and the virtual mass}

Let a resultant force $F_{R(i)}>0$ act on a train of a mass $m$, which moves at a velocity $v_{\mathrm{i}}$ at the start of the unit spacing $l_{i}=1, \forall i$. This force, which acts in the opposite direction of the direction in which the train is moving, can be the resultant force of braking produced by: a single braking system or by breaking systems; by the braking system and resistance; or by more than one type of resistance.

Under the conditions stated above, the resultant force will cause the deceleration of the train $a_{i}$, according to Newton's law. At the end of the unit spacing $l_{i}$, a change in the velocity $\Delta v=v_{i}-v_{i+1}$ occurs due to the action of the force. The momentum change caused by the resultant force $F_{R(i)}>0$ is equal to:

$$
\Delta K=v_{i} \cdot m_{t}-v_{i+1} \cdot m
$$

If the change in momentum is described by the theory of perfectly elastic central collision using the model of virtual masses at rest, we obtain the value of virtual mass which has caused the change in momentum $\Delta K$ at unit spacing:

$$
m_{i}^{\prime}=m_{t} \cdot \frac{v_{i}-v_{i+1}}{v_{i}+v_{i+1}}
$$

Introducing the deceleration $a_{i}$ at a length of the path travelled $l_{i}$, we obtain:

$$
m_{i}^{\prime}=m \frac{v_{i}-\left(v_{i}-\frac{a_{i} \cdot l_{i}}{v_{i}}\right)}{v_{i}+\left(v_{i}-\frac{a_{i} \cdot l_{i}}{v_{i}}\right)}=m \frac{\frac{a_{i} \cdot l_{i}}{v_{i}}}{\frac{2 \cdot v_{i}^{2}-a_{i} \cdot l_{i}}{v_{i}}}=\frac{F_{R(i)} \cdot m \cdot l_{i}}{2 \cdot v_{i}^{2} \cdot m-F_{R(i)} \cdot l_{i}}
$$


As $l_{i}=1$, we obtain the value of virtual mass at unit spacing and the force which is equivalent to the action of the virtual mass at unit spacing:

$$
m_{i}^{\prime}=\frac{F_{R(i)} \cdot m}{2 \cdot v_{i}^{2} \cdot m-F_{R(i)}}, F_{R(i)}=\frac{2 \cdot v_{i}^{2} \cdot m_{i}^{\prime} \cdot m}{m+m_{i}^{\prime}}
$$

If a resultant force $F_{R(i)}$ that is opposite to the direction of the motion acts on a train of mass $m$ on a length of path travelled $l_{i}$, the deceleration $a_{i}$ caused by that force is equal to the deceleration that would be caused by a perfectly elastic collision between the train and the virtual mass $\mathrm{m}^{\prime}$ which is in the state of rest on the line along which the train moves.

The basic types of resistance include the friction in the bearings on axles, the resistance of the wheel rolling on railway tracks, the resistance of the wheel sliding on rails, the resistance due to rail joints, the resistance in the joints between carriages, the resistance of pantograph, air resistance, etc. The value of the sum of basic resistances as a function of the train speed is expressed by a second-order polynomial, which was established a long time ago by Schmidt (1910), Strahl (1913), Davis (1926), Munkhacev (1927) and proved by Muhlenberg [1] and Mancini et al. [16] and Lukaszewicz [17, 18]:

$$
F=A+B v+C v^{2}
$$

We may now pose the question whether it is possible to separate air resistances from this basic expression for calculating the sum of basic resistances by using the virtual mass model. The idea comes from the fact that air resistances are a consequence of the action of one material system (air), and all other resistances involved are a consequence of another material system (ground, i.e. gravity).

One possible solution is to calculate the limit values of virtual masses obtained from the changes in kinetic energy at an infinite train speed.

Theorem: If the function of the train resistance is described by a standard second-order polynomial with the values of $A, B$ and $C$ expressing the speed in $\mathrm{m} / \mathrm{s}$, then, at infinite train speed, a half of coefficient $C$ is equal to the value of virtual mass at a unit distance.

Proof: In the case of the action of resistance, the mean train speed, the mean resistance force at a unit distance and consecutive changes in kinetic energy at the unit distance $(i, i+1)$ are:

$$
\begin{gathered}
v_{(i, i+1)}=\frac{v_{i}+v_{i+1}}{2}, \quad F_{(i, i+1)}=A+B v_{(i, i+1)}+C v_{(i, i+1)}^{2}, \\
E_{k(i+1)}=E_{k(i)}-F_{(i, i+1)} \Delta l
\end{gathered}
$$


Let us express the value of virtual mass as a function of a constant resistance force by using expression (11). The change in kinetic energy at a unit distance, obtained by replacing the speeds and masses, is equal to:

$$
\begin{aligned}
& \frac{m v_{i+1}^{2}}{2}=\frac{m v_{i}^{2}}{2}-A \Delta l-B \Delta l \frac{v_{i}+v_{i+1}}{2}-C \Delta l\left(\frac{v_{i}+v_{i+1}}{2}\right)^{2} \Leftrightarrow \\
\Leftrightarrow & 2 m v_{i+1}^{2}=2 m v_{i}^{2}-\Delta l\left(4 A+2 B v_{i}+2 C v_{i+1}+C v_{i}^{2}+2 C v_{i} v_{i+1}+C v_{i+1}^{2}\right) \Leftrightarrow \\
\Leftrightarrow & v_{i+1}^{2}(2 m+C \Delta l)+v_{i+1} \Delta l\left(2 B+2 C v_{i}\right)-v_{i}^{2}(2 m-C \Delta l)+\Delta l\left(2 B v_{i}+4 A\right)=0
\end{aligned}
$$

By solving the above quadratic equation, one can obtain the value of speed $v_{i+1}$ at the end of the path travelled. The velocity $v_{i+1}$ is a function of the speed $v_{i}$ and the action of the mean value of constant resistance forces $F_{(i, i+1)}$ :

$$
\begin{aligned}
& v_{i+1}=\frac{-\Delta l\left(2 B+2 C v_{i}\right) \pm \sqrt{D}}{2(2 m+C \Delta l)} \\
& D=2 \Delta l\left(B-C v_{i}\right)^{2}+4(2 m+C \Delta l)\left(v_{i}^{2}(2 m-C \Delta l)-2 \Delta l\left(B v_{i}-A\right)\right)
\end{aligned}
$$

If we now insert this value into expression (8) to calculate the values of virtual mass and if we find the limit value of the virtual mass when the velocity tends to infinity, there are only the terms with the highest exponent of the velocity $v_{i}$ which remain:

$$
\begin{gathered}
\Rightarrow \lim _{v_{i} \rightarrow \infty} m^{\prime}=\lim _{v_{i} \rightarrow \infty}\left(m \cdot \frac{v_{i}-v_{i+1}}{v_{i}+v_{i+1}}\right)= \\
=m \cdot \frac{1-\frac{-2 \Delta l C \pm \sqrt{4 \Delta l C^{2}+4(2 m+\Delta l C)(2 m-\Delta l C)}}{2(2 m+\Delta l C)}}{1+\frac{-2 \Delta l C \pm \sqrt{4 \Delta l C^{2}+4(2 m+\Delta l C)(2 m-\Delta l C)}}{2(2 m+\Delta l C)}} \\
=m \cdot \frac{4 m+4 \Delta l C \mp \sqrt{4 \Delta l C^{2}+16 m^{2}-4 \Delta l C^{2}}}{4 m \pm \sqrt{4 \Delta l C^{2}+16 m^{2}-4 \Delta l C^{2}}}= \\
=m \cdot \frac{4 m+4 \Delta l C \mp 4 m}{4 m \pm 4 m}
\end{gathered}
$$

In a central collision between the train and the virtual mass, the backward motion of the train cannot happen as the train mass is incomparably greater than the unit virtual mass. The impulse of force in the described system is always positive; therefore, only the real solution from expression (15) can be accepted, which means that the sign "-" from the numerator and the sign "+" from the 
denominator are taken into account (these two signs are appropriate for the solution 0 to the quadratic equation). Thus we get that the value of virtual mass at an infinite speed is equal to:

$$
\lim _{v_{i} \rightarrow \infty} m^{\prime}=m \cdot \frac{4 \Delta l C}{8 m}=\frac{\Delta l C}{2} \Leftrightarrow m^{\prime}=\frac{C}{2} \Leftrightarrow 2 m^{\prime}=C
$$

In the virtual mass model, the virtual mass disappears after the train collides with it. In real conditions, after the train collides with the real mass air molecules, the air molecules do not disappear and they react with other air molecules in a series of collisions. However, in the expressions for the calculation of basic resistances, in which air resistances are included, real features and relations of the collision between air molecules and the train are included. These impacts can be transferred to the virtual mass.

In consecutive collisions, the distribution of virtual masses on the path of the train constitutes the linear density of virtual masses measured in $(\mathrm{kg} / \mathrm{m})$. The product of the coefficient $C$ and the square of velocity is equal to the force $F$, i.e. $C \cdot v^{2}=F$.

The part of the product relating to basic resistances is in the SI because the linear density of virtual masses is expressed in the $(\mathrm{kg} / \mathrm{m})$ unit. Expression (16) is dimensionally in accordance with that. With an increase in speed, the value of virtual mass $m^{\prime}$ converges to a half of the coefficient $C$ from the basic expression for the train resistance calculation (11).

As the train moves through the air medium at rest, the volume of the air is determined by the product of unit spacing and the frontal area of the train and it has the same mass. If the mass of air from the defined volume is conceived as one whole, we obtain the linear density of virtual masses. According to the above developed theorem, this imaginary mass has a quantity of a half of the $C$ coefficient from expression (11), as shown in Figure 2.

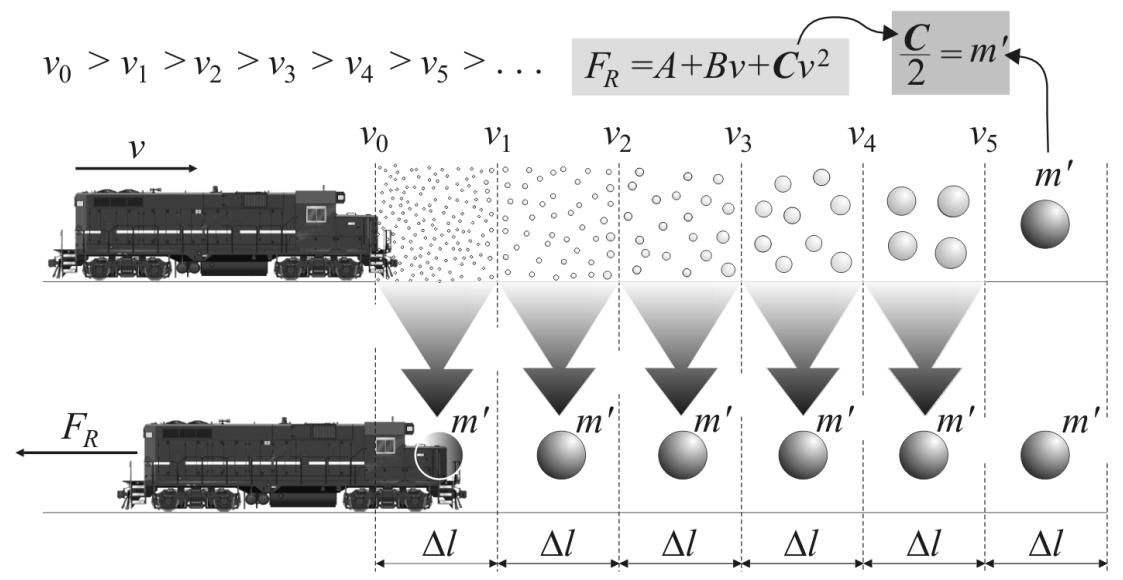

Figure 2: A virtual mass model. 


\section{Relation between the virtual mass and aerodynamic drag coefficient $C_{D}$}

Aerodynamic drag, the part which is dependent upon speed squared, is usually written for no wind conditions as:

$$
F_{D}=\frac{1}{2} \rho A_{f} C_{D} v^{2}=C v^{2} \Leftrightarrow \frac{1}{2} \rho A_{f} C_{D}=C
$$

where $\rho$ is the density of air, $A_{f}$ is the frontal area of train, $C_{D}$ is the aerodynamic drag coefficient [19]. If the left side of equation (17) is expanded with a unit quotient of the length of the train $L_{T}$, the volume of the train $V_{T}$ is equal to $A_{f} L_{T}$. The relation between the coefficient $C$ and the virtual mass, according to (16) and (17), establishes a relation between the aerodynamic drag coefficient $C_{D}$ and the virtual mass $m^{\prime}$ :

$$
\begin{gathered}
\frac{1}{2} \rho A_{f} L_{T} \frac{C_{D}}{L_{T}}=C=2 m^{\prime} \\
m^{\prime}=\frac{\rho A_{f} C_{D}}{4}=\frac{\rho A_{f} L_{T} C_{D}}{4 L_{T}}=\frac{\rho A_{f} V_{T} C_{D}}{4 L_{T}}
\end{gathered}
$$

The form of this expression is the basis for the interpretation of virtual mass which is proportional to one quarter of the mass of the air which is displaced by one meter of the train. The coefficient of proportion is the aerodynamic drag coefficient $C_{D}$ (Fig. 3).

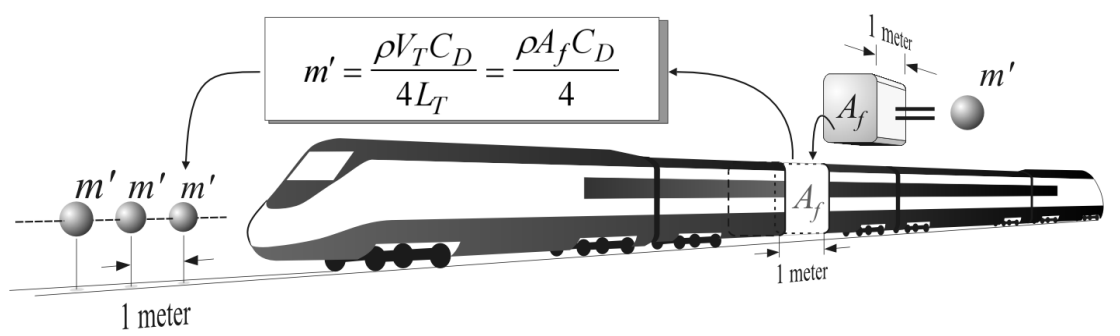

Figure 3: Interpretation of virtual mass.

In addition, the aerodynamic drag coefficient $C_{D}$ can also be interpreted as a quotient of a quadruple value of virtual mass and the mass of the air displaced by the train at a unit spacing of 1 meter.

$$
m^{\prime}=\frac{\rho A_{f} C_{D}}{4} \Leftrightarrow C_{D}=\frac{4 m^{\prime}}{\rho A_{f}}
$$

With relation (16), (18) and (20), final expression for aerodynamic drag coefficient $C_{D}$ calculation is: 


$$
C=2 m^{\prime} \Leftrightarrow C_{D}=\frac{4 m^{\prime}}{\rho A_{f}}=\frac{2 C}{\rho A_{f}}
$$

\section{Practical application of virtual masses theory}

We are going to examine how linear density value changes depending on changes of contents length and mass. Then the front and top surfaces of train remain of the same in shape, whereas side surface increases. This example is examined on ETR 500 with the following characteristics [16]:

- The mass of a locomotive is $67.2 \mathrm{t}$ and a carriage mass is $44.5 \mathrm{t}$;

- The length of a locomotive is $20.46 \mathrm{~m}$ with cross-section $12.800 \mathrm{~m}^{2}$,

- The length of a carriage $26.10 \mathrm{~m}$ with cross-section $10.868 \mathrm{~m}^{2}$;

Experimental measuring revealed the following basic resistance formula (for a composition of two locomotives and $n$ carriages, the speed is in $\mathrm{km} / \mathrm{h}$ ) with non-integrated bogies [16] is:

$$
F=(1.51+0,638 n)+(2.71+0.560 n) v_{t}^{2} 10^{-4}(\mathrm{kN})
$$

For speed in $\mathrm{m} / \mathrm{sec}$ and resistance in $(\mathrm{N})$, resistance formula is:

$$
F=1510+638 n+(3.516+0.7257 n) v_{t}^{2}(\mathrm{~N})
$$

The presentation of calculated virtual mass values for ETR500 train with two locomotives and $n$ carriages for non-integrated bogies is revealed in table 1 . Aerodynamic drag coefficient $C_{D}$ is calculated according to (21), value for density of air is $1.225 \mathrm{~kg} / \mathrm{m}^{3}$ (at sea level and at $15^{\circ} \mathrm{C}$ according to ISA International Standard Atmosphere) and $A_{f}$ is average frontal area (cross section).

Table 1: Calculation of virtual mass value at a unit distance and aerodynamic drag coefficient for different ETR 500 train configurations.

\begin{tabular}{|c|c|c|c|c|c|c|c|}
\hline $\begin{array}{c}\text { No. of } \\
\text { carr. }\end{array}$ & $\begin{array}{c}\text { Mass } \\
(\mathrm{t})\end{array}$ & $\begin{array}{c}L_{t} \\
(\mathrm{~m})\end{array}$ & $\begin{array}{c}A_{f} \\
\left(\mathrm{~m}^{2}\right)\end{array}$ & $A$ & $C$ & $m=\frac{C}{2}$ & $C_{D}$ \\
\hline 0 & 134.4 & 40.92 & 12,80 & 1510 & 3,51 & 1,756 & 0,2239 \\
\hline 1 & 178,9 & 67,02 & 12,16 & 2148 & 4,23 & 2,118 & 0,2843 \\
\hline 2 & 223,4 & 93,12 & 11,83 & 2786 & 4,96 & 2,481 & 0,3424 \\
\hline 3 & 267,9 & 119,22 & 11,64 & 3424 & 5,68 & 2,844 & 0,3989 \\
\hline 4 & 312,4 & 145,32 & 11,51 & 4062 & 6,41 & 3,207 & 0,4549 \\
\hline 5 & 356,9 & 171,42 & 11,42 & 4700 & 7,14 & 3,570 & 0,5103 \\
\hline 6 & 401,4 & 197,52 & 11,35 & 5338 & 7,86 & 3,933 & 0,5657 \\
\hline 7 & 445,9 & 223,62 & 11,30 & 5976 & 8,59 & 4,296 & 0,6206 \\
\hline 8 & 490,4 & 249,72 & 11,25 & 6614 & 9,31 & 4,659 & 0,6761 \\
\hline 9 & 534,9 & 275,82 & 11,22 & 7252 & 10,04 & 5,021 & 0,7306 \\
\hline
\end{tabular}


Physical air resistance interpretation on the example of a train set consisting of two engines and 9 carriages with non-integrated bogies equals serial run of the train front against virtual masses of $5.021 \mathrm{~kg}$ on every meter of crossed route.

By adding one carriage with non-integrated bogies the virtual mass is always increased by $0.3628 \mathrm{~kg} / \mathrm{m}$ per one carriage. The difference represents the virtual masses linear density increase arising from adding one inter-carriage without front and rair. Aerodynamic drag coefficient $C_{D \text { (carr) }}$ for one carriage with non-integrated bogies side surface, without front and rair is:

$$
C_{D(\text { carr })}=\frac{2 \cdot 0.3628}{1.225 \cdot 10.868}=0.0545
$$

\section{Conclusion}

Conditions of the virtual mass convergence at an infinite speed are required only for the creation of the virtual mass model. In the zones of high speeds, with the domination of air resistance, the virtual mass is equal to the theoretically proved value of a half of the coefficient $C$ multiplied by the speed squared. At low speeds, a set of gravitational resistances is dominant in the resistance resultant. The virtual mass has the same theoretical value, but its participation in the value of total train resistance is negligible. In practical applications, the virtual mass is the invariance of the speed and it is always equal to $C / 2$.

If the train moves through the air in no wind conditions, the virtual mass need not have a constant value and a stationary linear density. The change in the volume that occurs as the train enters a tunnel increases the value of virtual mass.

The virtual mass model has a special advantage in the possibility of calculating partial aerodynamic characteristics such as the resistance of the side surfaces of the train when the front/rear surfaces and the vertex surfaces are blocked by other carriages of the train.

The sources of variations in the value of virtual mass are multiple. The virtual mass model can also be applied in the case of wind conditions. All possible variations of the values of virtual mass can easily be quantified by the resistance force of the air.

\section{Symbols}

$a$ - acceleration (deceleration), $\mathrm{m} / \mathrm{s}^{2}$

$A_{f}$ - frontal area of the train, $\mathrm{m}^{2}$

$C_{D}$ - aerodynamic drag coefficient

$\Delta K-$ momentum change, $\mathrm{kg} \cdot \mathrm{m} / \mathrm{s}$

$A, B, C$ - coefficients of resistant function

$E_{k}-$ kinetic energy, $\mathrm{J}$

$m$ - mass of the system, mass of the train, $\mathrm{kg}$

$F$ - resistance force, $\mathrm{N}$

$m^{\prime}-$ mass of the system, virtual mass, $\mathrm{kg}$

$F_{D}$ - aerodynamic drag, $\mathrm{N}$

$\kappa-$ Newton's coefficient of restitution

$i-$ index of unit spacing

$F_{R}-$ resultant force, $\mathrm{N}$

$v_{t}-$ velocity of the train, $\mathrm{km} / \mathrm{h}$ or $\mathrm{m} / \mathrm{s}$

$\Delta l$ - unit spacing, $\mathrm{m}$

$L_{T}$ - length of the train, $\mathrm{m}$

$v, v^{\prime}-$ velocity of the virtual mass, $\mathrm{m} / \mathrm{s}$

$\rho-$ density of air, $\mathrm{kg} / \mathrm{m}^{3}$

$v_{i,}, v_{i}{ }^{\prime}-$ velocity of the virtual mass, $\mathrm{m} / \mathrm{s}$

$V_{T}$ - volume of the train, $\mathrm{m}^{3}$ 


\section{Acknowledgement}

The study is part of the investigations realised with the scope of the Project No. 36012 financially supported by the Ministry of Science and Technological Development of the Republic of Serbia.

\section{References}

[1] Schetz, J. A., Aerodynamics of high-speed trains, Annual Review of Fluid Mechanics. 33, pp. 371-414, 2001.

[2] Heine, C. and Matsche, G., The Influence of the Nose Shape of High Speed Train on the Aerodynamic Coefficients, Proceedings of the World Congress on Railway Research. Köln, 2001.

[3] Paradot, N. and Talotte, C., High Speed Train Running Resistance Analysis through Numerical Simulation and Experimental Investigation, Proceedings of the World Congress on Railway Research. Köln, 2001.

[4] Krajnovic, S., Improvement of aerodynamic properties of high-speed trains by shape optimization and flow control. Proceedings of the World Congress on Railway research. Seoul, 2008.

[5] Krajnovic, S., Shape optimization of high-speed trains for improved aerodynamic performance, Proc. IMechE, Part F: J. Rail and Rapid Transit. 223, pp. 439-452, 2009.

[6] Kwon, H., Park, Y., Lee, D., and Kim, M., Wind tunnel experiments on Korean high-speed trains using various ground simulation techniques, $J$. Wind Eng. Ind. Aerodyn. 89, pp. 1179-1195, 2001.

[7] Kim, D., H. and Shin, M., H., The Aerodynamic Effect of Air-shafts in the Single Tunnel with Small Cross Sectional Area on Conventional Line, Proceedings of the World Congress on Railway Research. Köln, 2001.

[8] Noger, C., Regardin, C. and Széchényi, E., Investigation of the transient aerodynamic phenomena associated with passing manoeuvres, Journal of Fluids and Structures, 21, pp. 231-241, 2005.

[9] Diedrichs, B., Berg, M., Stichel, S. and Krajnovic, S., Vehicle dynamics of a high-speed passenger car due to aerodynamics inside tunnels, Proc. IMechE, Part F: J. Rail and Rapid Transit. 221, pp. 527-545, 2007.

[10] Khier, W., Breuer, M. and Durst, F., Flow structure around trains under side wind conditions: a numerical study, Comput. Fluids. 29, pp. 179-195, 2000.

[11] Suzuki, M., Tanemoto, K. and Maeda, T., Aerodynamic characteristics of train/vehicles under cross winds, J. Wind Eng. Ind. Aerodyn., 91, pp. 209218, 2003.

[12] Diedrichs, B., On computational fluid dynamics modelling crosswind effects for high-speed rolling stocks, Proc. IMechE, Part F: J. Rail and Rapid Transit. 217, pp. 203-226, 2003. 
[13] Sanquer, S., Barré, C., Dufresne de Virel, M., and Cléon, L. M., Effect of cross winds on high-speed trains: development of a new experimental methodology, J. Wind Eng. Ind. Aerodyn., 92, pp. 535-545, 2004.

[14] Carrarini, A., Reliability based analysis of the crosswind stability of railway vehicles, J. Wind Eng. Ind. Aerodyn., 95, pp. 493-509, 2007.

[15] Bocciolone, M., Cheli, F., Coradi, R., Maggiasaca, S. and Tomasini, G., Crosswind action on rail vehicles: Wind tunnel experimental analysis, $J$. Wind Eng. Ind. Aerodyn. 96, pp. 584-610, 2008.

[16] Mancini, G., Malfatti, A., Violi, A. G. and Matschke, G., Effects of Experimental Bogie Fairings on the Aerodynamic Drag of the ETR 500 High Speed Train, Proceedings of the World Congress on Railway Research. Köln, 2001.

[17] Lukaszewicz, P., A simple method to determine train running resistance from full-scale measurements, Proc. IMechE, Part F: J. Rail and Rapid Transit, 221, pp. 331-337, 2007.

[18] Lukaszewicz, P., Running resistance - results and analysis of full-scale tests with passenger and freight trains in Sweden, Proc. IMechE, Part F: J. Rail and Rapid Transit, 221, pp. 183-192, 2007.

[19] Lukaszewicz, P., Running resistance and energy consumption of ORE trains in Sweden, Proc. IMechE, Part F: J. Rail and Rapid Transit, 223, pp. 189-197, 2009. 